\title{
Temporal Evolution of Roughness Development on Polymer Surfaces Exposed to Non-Thermal Plasma
}

\author{
Taís Felix, ${ }^{\oplus a, b}$ Luís O. B. Benetoli, ${ }^{a}$ Susana Sério, ${ }^{b}$ Marta E. R. Dotto ${ }^{c}$ and \\ Nito A. Debacher ${ }^{(1)} * a$ \\ ${ }^{a}$ Departamento de Química, Universidade Federal de Santa Catarina, \\ 88040-900 Florianópolis-SC, Brazil \\ ${ }^{b}$ CEFITEC, Departamento de Física, Faculdade de Ciências e Tecnologia, \\ Universidade Nova de Lisboa, 2829-516 Caparica, Portugal \\ 'Departamento de Física, Universidade Federal de Santa Catarina, \\ 88040-900 Florianópolis-SC, Brazil
}

\begin{abstract}
Processes such as ablation, erosion and corrosion generally create roughness patterns on solid surfaces. Despite the randomness of this phenomenon, some patterns can be described mathematically by studying statistically the mechanisms behind them. This paper reports an experimental study on the temporal evolution of the roughness development of polymer surfaces exposed to argon non-thermal plasma under reduced pressure of 5 mbar and $65 \mathrm{~W}$ of applied power. The substrates studied were polypropylene, high density polyethylene, polyamide- 6 and poly(ether ether)ketone. The mean roughness data as well as the saturation roughness values showed differences between polyolefin and polymers containing heteroatoms and notably the dependence of the etching on the packing density of the polymer chains. The plasma-etched surfaces were described statistically as self-affine surfaces using scaling law analysis, exhibiting roughness exponents of $\alpha$ ca.0.73 \pm 0.2 and growth exponents of $\beta$ ca. $1.0 \pm 0.1$. The roughness increases and decreases successively during treatment although presenting a general linear behavior in a non-monotonous way, as a function of time. The models for the shadowing and redistribution of active species can be complemented with the transient crosslinking model of the observed flattening stages.
\end{abstract}

Keywords: roughness surface, scaling laws, non-thermal plasma, polymer surface

\section{Introduction}

The roughness of an ordinary polymeric surface, natural or synthetic is an important feature that modulates the interactions between the medium and the interface. The magnitude of this property defines the tendency of surface adhesion which has important applications, for instance in heterogeneous catalysis, electrochemistry and biology. ${ }^{1}$

Increasing the roughness of a surface or inducing the surface roughness development or growth in the form of electrochemical deposition, erosion, corrosion or the growth of films or crystals, may result in different flat, facetted, pointed or disordered forms and complex structures. ${ }^{2}$ Although estimating the roughness of a surface and the surface energy is the key to understanding surface phenomena, in general, in terms of its application, a surface

*e-mail: nito.debacher@ufsc.br is considered to be flat and smooth. Concepts of fractal geometry ${ }^{3}$ and other tools associated with statistical physics, such as the applied scaling law have proved to be efficient to describe rough surfaces and have therefore become the object of theoretical and experimental studies. ${ }^{4-6}$

These processes and the growth phenomena are studied using statistical mechanics, which is used to develop and explain macroscopic and mesoscopic laws for microscopic systems. ${ }^{7}$ Since the properties of a thin-film are related to its microstructure, especially to its morphology and surface roughness, ${ }^{8}$ the growth of a thin-film surface, or simply, surface growth can be evaluated by a dynamical study.

The non-thermal plasma (NTP) treatment can change physical and/or chemical properties of solid surfaces, which is useful in several technological areas such as subtractive microfabrication, photolithography, superhydrophilic surfaces, adhesion promotion, friction coefficient and coating processes with the deposition of active or protective 
films, as well as chemical functionalization. The collisional events that occur as the species generated by the plasma interact with the surface can lead to roughness between 0.2-5 $\mu \mathrm{m}$ in polymer films, among other materials ${ }^{9}$ and statistical models need to be developed in order to explain this behavior mathematically. During surface growth, it is possible to establish relationships between roughness data and exposure time from topographic patterns obtained by image analysis fractioning the sample width into matrix subdivisions. The results obtained enable the definition of a growth model for a particular system and it can be related to a universality class, as described by the fractal concept of surface growth using a scaling law, as previously noted by Edwards-Wilkinson. ${ }^{10,11}$ Models of growth phenomena are very powerful tools that can help researchers to predict and control physical, chemical, and mechanical properties. ${ }^{8}$

For practical purposes, understanding this concept helps the optimization of NTP surface exposure by predicting the time limit to obtain the appropriate roughness profiles. At surface growth process by NTP, discharge active species are continuously produced and consumed; therefore, a steadystate regime can be considered. In contrast, in conventional chemical attack, the chemical potential decreases over time and the efficiency is therefore, limited. ${ }^{12,13}$ The roughness profile produced by chemical attack has been reported by several researchers, ${ }^{14-16}$ however, the literature is relatively scarce, ${ }^{17,18}$ particularly for the cases where the sputter etching agent is a non-thermal plasma. ${ }^{19-22}$

Authors have proposed interesting surface growth models, when studying particular polymeric systems and the influence of NTP on the formation of roughness. Bruce et al..$^{23}$ studied polystyrene films assuming that the formation of surfaces is the cause of ion-bombardment and, under the effect of temperature, the surface presents a wrinkle behavior and microscale reticulations. Some authors, ${ }^{24-27}$ however, named the observed height differences as nanodots and related their formation to a synergistic effect between the ion bombardment and the deposition of etch inhibitors.

According to the fractal concept of surface growth, ${ }^{10}$ the bombardment of active species occurs randomly, and the peaks will be more affected than the valleys on a previously rough surface. Surface diffusion will therefore occur, minimizing these heights and polishing the surface. The deposition models can be applied to sputter etching processes; however it must be considered that the species originating from the plasma, including radiation, penetrate into the inner layers of the material, causing changes in the bonds and consequently in the integrity of the substrate.

Thus, the depth to which these species can penetrate the surface and the angle of incidence of the plasma can affect the values for the roughness $(\alpha)$ and growth $(\beta)$ exponents considered in the scaling laws. This model was used by Pétri et al., ${ }^{28}$ to explain their observations, referred to as "shadowing" or shaded growth. In this etching process, there is a rapid erosion of the peaks thereby "softening" the surface, leading to a growth parameter of around $\beta=1$.

Interestingly, a further increase in roughness is observed empirically after the surface has reached a low relief stage. To address this, a second approach was proposed by Zhao et al. ${ }^{29}$ who modified silicon surfaces (100) by etching with $\mathrm{CF}_{4}$ plasma. The authors obtained roughness and growth exponents of around $0.96 \pm 0.06$ and $0.91 \pm 0.03$, respectively, and proposed a model for the redistribution of the active species on the surface. In this regard, Drotar et al., ${ }^{30}$ used Monte Carlo simulations to define a new universality class where $\alpha$ ca. $\beta$ ca. z. ca. 1 . According to this model, the roughness remains in constant evolution due to the "late" collisions of the species that interact with the surface. In a similar study, Agarwal et al., ${ }^{31}$ found that there is a limit roughness value at which the growth profile occurs in a sublinear, irregular way, where $\beta$ ca. 0.5 can represent a random behavior. Above this limit value, the roughness starts to follow a linear course, increasing with time and presenting $\beta=1 .^{31}$

Experimentally, to determine the parameter of surface roughness development or growth $(\alpha)$, images are obtained using atomic force microscopy (AFM). The images are then statistically analyzed with regard to the topographic variation (valleys and peaks presence) by fractioning the sample width into matrix subdivisions. The statistical analysis of the data using the scaling law can be performed considering the width of the interface (W), defined as the height $(\mathrm{h})$ of fluctuations in a matrix of size $\mathrm{L}$, which is calculated through equation 1 :

$\mathrm{W}(\mathrm{L}, \mathrm{t})=\sqrt{\frac{1}{\mathrm{~L}} \sum_{\mathrm{i}=1}^{\mathrm{L}}[\mathrm{h}(\mathrm{i}, \mathrm{t})-\overline{\mathrm{h}}(\mathrm{t})]^{2}}$

This equation allows us to determine the roughness exponent $(\alpha)$. Thus,

$\mathrm{W}(\mathrm{L})$ ca. $\mathrm{L}^{\alpha} \rightarrow \log \mathrm{W}(\mathrm{L})$ ca. $\alpha \log \mathrm{L}$

The log-log graphs of $\mathrm{W}(\mathrm{L})$ as a function of $\mathrm{L}$ provide a curve with two distinct regions: a self-affine (slope) region, where $\mathrm{W}(\mathrm{L})$ is dependent on $\mathrm{L}$, and a saturation region, where $\mathrm{W}(\mathrm{L})$ is independent of $\mathrm{L}$. The interface between the self-affine and saturation regions is defined as the correlation length ( $\mathrm{Lc}$ ), which provides information on how the surface is correlated. If $\mathrm{L}$ is greater than $\mathrm{Lc}$, the whole surface is correlated, so Lc is the typical distance from one known height to a neighboring height. ${ }^{10,12}$ 
The roughness exponent $(\alpha)$ is obtained from the plot of interface width (W) as a function of length (L-size of matrix). The curve of $\mathrm{W} \times \mathrm{L}$ plot shows two regions: the first-slope region-to small $\mathrm{L}<\mathrm{Lc}$ is the linear region where $\mathrm{W}$ scales with $\mathrm{L}$ by a factor $\alpha$. The second region to $\mathrm{L}>\mathrm{Lc}$ that is saturation region-where $\mathrm{W}$ has no dependence on $\mathrm{L}$-constant-and is called saturation roughness, $\mathrm{W}_{\text {sat }}$, related to a fixed plasma exposure time. After this, to obtain growth exponent $(\beta)$ from each $\mathrm{W}_{\text {sat }}$ on time t of plasma exposure, is plotted $\mathrm{W}_{\text {sat }}$ as a function of the plasma exposure time, to which the samples were subjected. From the slope of de curve, $\beta$ is obtained, ${ }^{12,32}$ as described by equation bellow:

$\mathrm{W}_{\text {sat }}$ ca. $\mathrm{t}^{\beta} \rightarrow \log \mathrm{W}$ ca. $\beta \log \mathrm{t}$

Based on the above assumption the temporal evolution of roughness growth on polymer surfaces exposed to NTP can be described and the surface roughness can then be predicted according to the plasma exposure time. In this context, the aim of this study was to apply statistical analysis based on scaling laws to predict the time limit to obtain appropriate roughness profiles on polymer surfaces exposed to NTP based on surface micrograph analysis by AFM.

\section{Experimental}

\section{Polymers}

The polymers were chosen according to chemical structure, physical properties (Figure 1 and Table 1) and applications. High density polyethylene (HDPE) is classed as a polyolefin of non polar, saturated, high molecular weight hydrocarbons, commonly used in plastic bags, films and bottles. Polypropylene (PP) is also a polyolefin with branched carbonic chain with high chemical resistance widely used in packaging and labeling processes. The polyamide used (PA6.6 or nylon 66) is classed as aliphatic polyamides and due to their durability and strength are commonly used in many manufactured goods like textiles and sportswear. Polyether ether ketone (PEEK) is classed as polyketones with aromatic rings and excellent chemical resistance properties used in engineering applications. The four polymers were obtained on a donation basis, where PP,
Table 1. Physical properties of polymer samples ${ }^{33}$

\begin{tabular}{lccc}
\hline & $\begin{array}{c}\text { Crystalline } \\
\text { index / \% }\end{array}$ & $\begin{array}{c}\text { Density / } \\
\left(\mathrm{g} \mathrm{cm}^{-3}\right)\end{array}$ & $\begin{array}{c}\text { Dielectric } \\
\text { constant }(\varepsilon)\end{array}$ \\
\hline PP & 42.5 & 0.90 & 2.5 \\
HDPE & 49.5 & 0.98 & 2.35 \\
PA6.6 & 14.7 & 1.13 & 3.6 \\
PEEK & 33.5 & 1.26 & 3.3 \\
\hline
\end{tabular}

PP: polypropylene; HDPE: high density polyethylene; PA6.6: polyamide 6.6; PEEK: poly(ether ether)ketone.

HDPE and PA6.6 from Fortymil Plastics (Itatiba, Brazil) and PEEK from Modern Plastics (Shelton, USA).

These polymeric materials were heated on a Velp heating plate and molded to $150-200 \mu \mathrm{m}$ films by pressing over high heat resistance Kapton ${ }^{\circledR}$ film (Dupont, Kansas, USA). Then films were cooled naturally at room temperature. The films were then cut in pieces of $20 \times 20 \mathrm{~mm}$, washed with isopropanol (Hach, Lisboa, Portugal) in an ultrasonic bath and stored at room temperature in a desiccator.

\section{Dielectric barrier discharge (DBD)-NTP}

The samples were fixed on a $1.2 \mathrm{~mm}$ thick cylindrical borosilicate glass plate and centered on the lower electrode. The distance between the electrodes was set at $4 \mathrm{~mm}$, according to Figure 2. The reactor was operated with a gas pressure of 5 mbar using argon (99.99\%) and the radio frequency power source was set at $65 \mathrm{~W}$ and $450 \mathrm{kHz}$. The samples were exposed to a glow discharge regime for 5,10 , 20,30, 60, 80 and 100 min and then stored in individual plastic bags.

\section{Chemical composition analysis by X-ray photoelectron} spectroscopy (XPS)

The XPS analysis of the polymer samples were performed on unexposed and exposed to NTP for $10 \mathrm{~min}$ in order to evaluate the chemical composition of the surface. The measurements were carried out in a custom-built experimental setup, ${ }^{34}$ equipped with a VSW XPS system with the Class 100 energy analyzer, that uses the nonmonochromatic $\mathrm{Mg} \mathrm{K} \alpha$ line (photon energy of $1253.6 \mathrm{eV}$ ). The energy scale was calibrated to the peak position of the

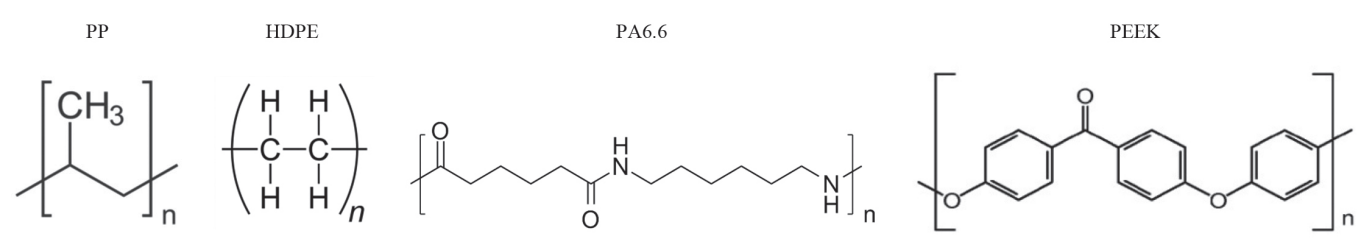

Figure 1. Skeletal structures of polymer samples used. 


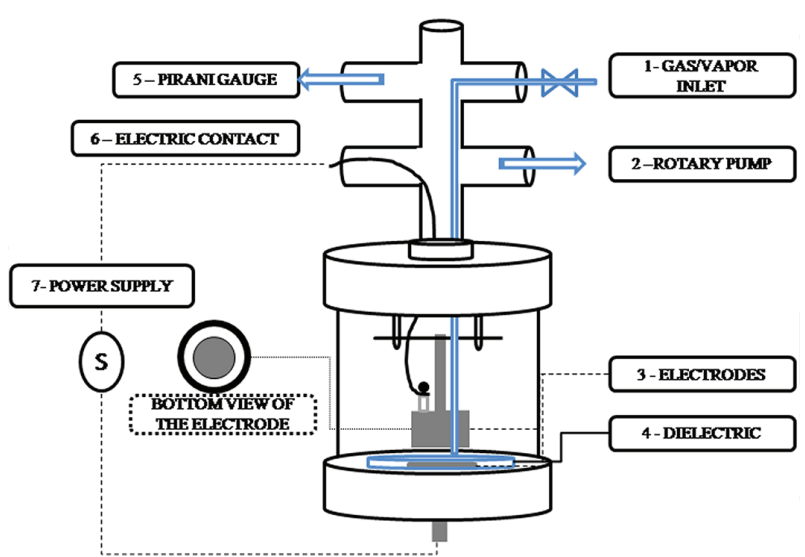

Figure 2. Schematic diagram of the NTP apparatus.

$\mathrm{Ag} 3 \mathrm{~d}_{5 / 2}(368.22 \mathrm{eV})$ and $\mathrm{Au} 4 \mathrm{f}_{7 / 2}(83.96 \mathrm{eV})$ lines using single crystal Ag (110) and a polycrystalline Au sample, respectively. Survey and high resolution spectra were taken in the Fixed Analyzer Transmission mode using the pass energy of $44 \mathrm{eV}$ (energy step/gate time of $0.5 \mathrm{eV} / 0.5 \mathrm{~s}$ ) and $22 \mathrm{eV}$ (energy step/gate time of $0.1 \mathrm{eV} / 4 \mathrm{~s}$ ), respectively. ${ }^{35}$

All analyses have been performed as-received, i.e., without any additional sample preparation (cleaning in solvents or sputter cleaning in vacuum). The composition analysis has been performed on the basis of the intensities of $\mathrm{O} 1 \mathrm{~s}$ and $\mathrm{C} 1 \mathrm{~s}$ in a standard way, using appropriate atomic sensitivity factors (ASFs). The authors used GL(30) pseudo-Voigt peak profile and the constraint that full width at half maximum (FWHMs) of all contributions in the frame of a line are the same, while no constraints were used for the peak positions.

Morphological characterization of the samples by scanning electron and atomic force microscopies

The polymeric samples were exposed to plasma treatment for 5, 20, 60 and $100 \mathrm{~min}$ and then analyzed by scanning electron microscopy (SEM) using a JEOL microscope, model JSM-6390LV, under electron beam, set at $8 \mathrm{kV}$ with a scale of $5 \mu \mathrm{m}$. All samples were studied by AFM using a Nanosurf FlexAFM microscope, operating in tapping mode, at a rate of $1.0 \mathrm{~Hz}$ with a resolution of $512 \times 512$ pixels. Micrographs were obtained in 3 different regions of each sample and 4 images were obtained within each region, with a lateral size of 40, 20 and $10 \mu \mathrm{m}$. In the first step, the images were analyzed using the software $\mathrm{WSxM}^{36}$ (version 5.0 Develop 7.0) to apply the scaling analysis. From the output graphs and the coefficient analysis the critical exponents were obtained. Experimentally this critical exponent is determined by evaluating how the saturation roughness values change as a function of time (equations 1, 2 and 3).

\section{Results and Discussion}

The XPS analysis of the polymer samples unexposed and exposed to NTP, in atomic percentage (at\%) of each chemical group are shown in the Table 2 .

As can be observed from Table 2, taking the unexposed samples as the reference for the XPS analysis regarding the oxygen groups, all exposed samples increased the concentration of oxygen on the polymer surface and these results are in agreement with the literature. ${ }^{37-41}$

The unexposed samples of HDPE and PP (Table 2) showed small amount of oxygen, about 0.05 and 0.08 in $\mathrm{O} / \mathrm{C}$ ratio, respectively, related to oxygen groups of $\mathrm{C}-\mathrm{O}$ in HDPE and $\mathrm{C}-\mathrm{O} / \mathrm{C}=\mathrm{O}$ in $\mathrm{PP}$. The $\mathrm{O} / \mathrm{C}$ ratio can be related to surface contamination or oxidation due to heating and molding process during samples preparation as those polymers has no oxygen on their chemical structure. The unexposed samples of PA6.6 and PEEK showed slightly higher amount of oxygen when compared to HDPE and PP samples, about 0.2 and 0.13 in $\mathrm{O} / \mathrm{C}$ ratio, respectively, which are related to oxygen groups as part of the original chemical structure.

The exposed sample of HDPE showed 0.25 in O/C ratio, which is the highest degree of oxidation obtained when compared to PA6.6, PEEK and PP. The exposed samples of PEEK and PP showed 0.20 and $0.15 \mathrm{O} / \mathrm{C}$ ratio, respectively, and PA6.6 showed 0.24 in the $\mathrm{O} / \mathrm{C}$ ratio, the lower degree of oxidation among the studied polymers samples.

Table 2. Amount of O $1 \mathrm{~s}$ and N 1s on the surface, expressed as O/C and N/C, respectively, of polymer samples unexposed and exposed to NTP during 10 min

\begin{tabular}{lccrcr}
\hline \multirow{2}{*}{ Sample } & \multicolumn{2}{c}{$\mathrm{O} / \mathrm{C} / \mathrm{at} \%$} & & N/C / at $\%$ \\
\cline { 2 - 3 } \cline { 5 - 5 } & Unexposed to plasma & Exposed to plasma & & Unexposed to plasma & Exposed to plasma \\
\hline PP & 0.08 & 0.15 & & 0.04 \\
HDPE & 0.05 & 0.25 & 0.12 \\
PA6.6 & 0.2 & 0.24 & \\
PEEK & 0.13 & 0.20 & \\
\hline
\end{tabular}

PP: polypropylene; HDPE: high density polyethylene; PA6.6: polyamide 6.6; PEEK: poly(ether ether)ketone; O/C: amount of oxygen in carbon relation; N/C: amount of nitrogen in carbon relation; at $\%$ : atomic percentage. 
The HDPE and PP exposed samples to NTP showed an increasing in oxygen concentration and some oxidation of the $\mathrm{C}-\mathrm{O}$ group to $\mathrm{C}=\mathrm{O}$. The PA6.6 exposed samples showed the $\mathrm{C}=\mathrm{O}$ group from its chemical structure and additional groups of $\mathrm{C}-\mathrm{OH}(64.76 \%)$ and $* \mathrm{O}-(\mathrm{C}=\mathrm{O})-\mathrm{C}(67 \%)$. The PEEK exposed samples showed an increase in the number of $\mathrm{C}-\mathrm{O} / \mathrm{C}=\mathrm{O}$ groups in general and reduction of $12.08 \%$ of the $* \mathrm{O}-(\mathrm{C}=\mathrm{O})-\mathrm{C}$ group and the formation of a new similar group of $\mathrm{O}-(\mathrm{C}=\mathrm{O} *)-\mathrm{C}(48.27 \%)$.

This result shows that the oxidation process is the main driving force to act on surface degradation. Although the XPS analysis were performed for samples exposed to NTP for $10 \mathrm{~min}$, an increase in oxidation will be expected for longer exposure and therefore an increase in the oxygen concentration. As can be observed from Table 2, taking the unexposed samples as the reference for the XPS analysis regarding the oxygen groups, all exposed samples increased the concentration of oxygen on the polymers surface which is in agreement with the literature..$^{42} \mathrm{~A}$ brief description of the main groups identified on the surface of polymers, exposed to $10 \mathrm{~min}$ by NTP, can be found in the Supplementary Information (SI) section (Table S1).

Figure 3 shows the SEM and AFM images of the PP, HDPE, PA6.6 and PEEK polymers treated by argon NTP and all polymers studied showed patches of different shades related to erosion, with growing and decrease roughness overtime during treatment. A similar result was observed by our group in a previous study ${ }^{37}$ on the morphology of polymer surfaces by oxygen NTP, in which polyethylene terephthalate (PET) showed a greater roughness in a shorter time when compared to PEEK and this was related to the hardness of the polymer structure. However, it is important to note that NTP can induce polymeric chain crosslinking thus stabilizing the surface and preventing further erosion. ${ }^{43}$

Figure 1 and Table 1 show the chemical structure and physical properties of the polymer samples used and based on a visual examination from Figure 3 the resistance to erosion of the materials tested can be ordered as: HDPE $<$ PP $<$ PA6. $6<$ PEEK.

Although it appears that each SEM image of the etching stage is unique, Figure 3 shows that this process occurs in consecutive stages. The images obtained by AFM and the profiles of the evolution of the roughness, corroborate this finding. The images show how the surface topography is altered during the period of plasma exposure, presenting successive stages of elevation (light spots) and relaxation (dark grey surface). With regard to the PP and HDPE samples, based on Figures 3A-3B and Figures $4 a-4 b$, as a function of exposure time, the following considerations can be made: (i) The highest peaks of the pure sample are first worn by the plasma ionized species, according to the model of shadowing. The sputter etching of the surface would preferentially acts on spots of less physical rigidity or which are not crosslinked.

(ii) The ionized species from the plasma continues to reach the surface and its morphology becomes more uniform since the crosslinking increases the organization of the chains and, consequently, the surface density. It is important to note the similarity of the peaks and their distances on treated surfaces after 5 min with those observed after 20 or $60 \mathrm{~min}$.

(iii) With the increase in the surface density, the resistance to etching increases and therefore, the process becomes slower. It can be seen in Figure 4 that the second roughness plateau was reached after 60 min of exposure only for PP. The surface valleys must concentrate the active ionized species, according to the model of reemission of species proposed by Zhao et al. ${ }^{29}$ and Drotar et al. ${ }^{30}$ As the plasma provides the active ionized species in a continuous process, valleys and peaks are continuously being created.

The polyamide contains $\mathrm{CH}, \mathrm{C}=\mathrm{O}, \mathrm{NH}$ and $\mathrm{HCN}=\mathrm{O}$ groups, which are propitious to crosslinking, but these groups can also be desorbed from the surface by chain scission, favoring physical etching as confirmed by the final roughness value of almost $270 \mathrm{~nm}$ after $100 \mathrm{~min}$, according to Figure $4 \mathrm{c}$. Among the materials tested, PEEK has the highest packaging density and crystallinity index (30\%) (Table 1) and is considered one of the most resistant polymeric materials currently used industrially.

For the PEEK sample (Figure 3D) the layer of roughness produced in the first stage is removed after approximately $35 \mathrm{~min}$ and the roughness growth of the second stage, starts only after $100 \mathrm{~min}$ of exposure, which is related to the resistance of this material to erosion.

Plasma discharge induces crosslinking in polymers through exposure to UV radiation. In the case of polymers with a linear chain, such as HDPE, PP and PA6.6, preferential chain scission and crosslinking occurs and PA6.6 is expected to show greater degradation than the polyolefins, because it contains - $\mathrm{NCO}-$ and - $\mathrm{CO}-$ groups. ${ }^{9}$

The polymers samples used in this work can be associated in two groups based on their chemicals structure, Table 1. The first group (PP and HDPE) are based on hydrocarbons structure with high values of crystallinity and low density. The surface roughness is smaller on HDPE, which may be due to its higher crystallinity and density compared to PP. The second group (PA6.6 and PEEK) have some $\mathrm{CO}$ and $\mathrm{CN}$ functional groups in their structure and low crystallinity and the high-density values. The surface roughness of PA6.6 is higher than on PEEK, which may be 
(A)
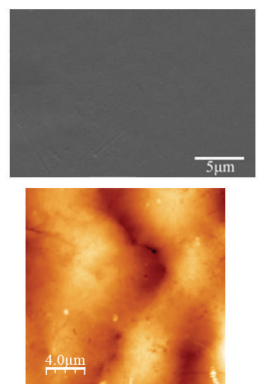

(a)
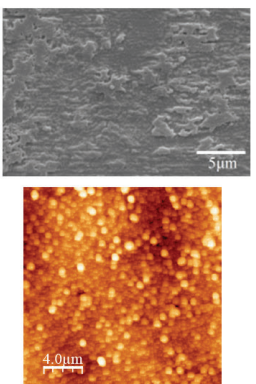

(b)
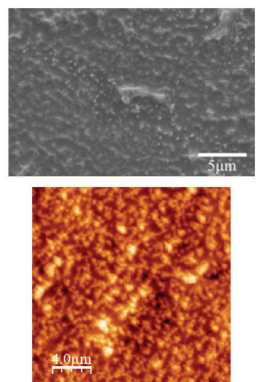

(c)


(d)
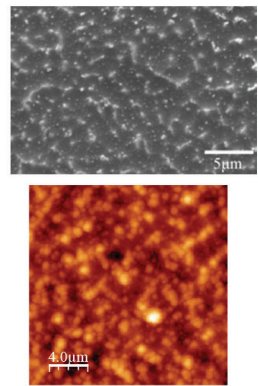

(e)
(B)
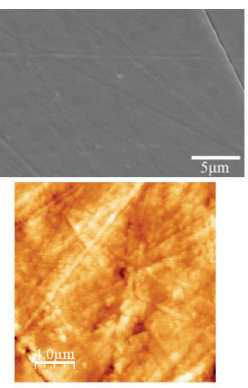

(a)
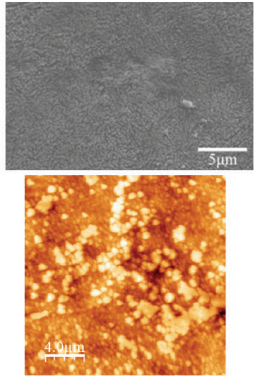

(b)
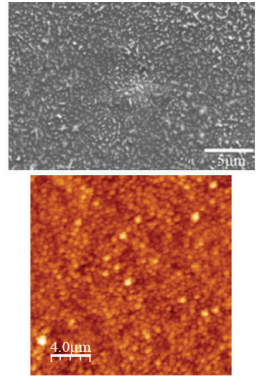

(c)
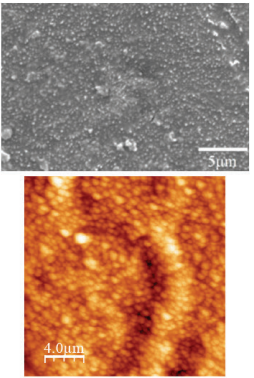

(d)
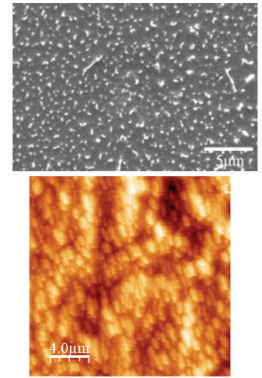

(e)
(C)
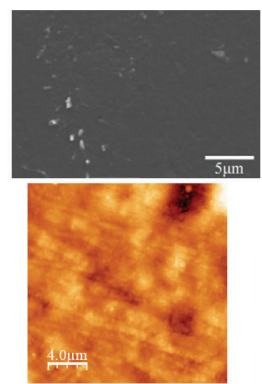

(a)
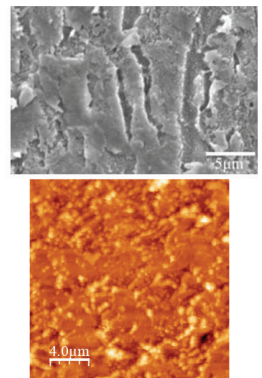

(b)

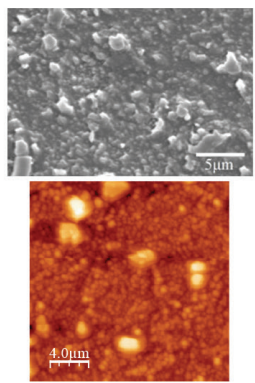

(c)

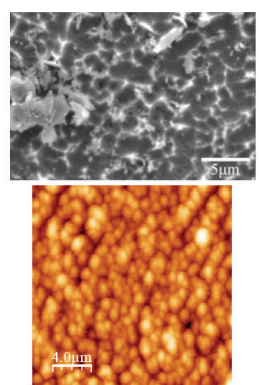

(d)

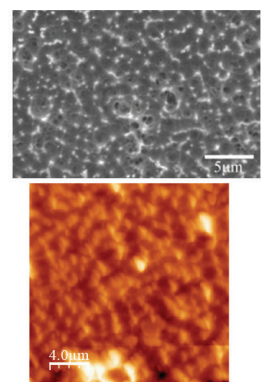

(e)
(D)
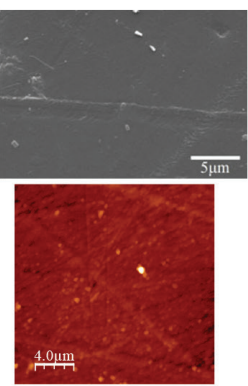

(a)
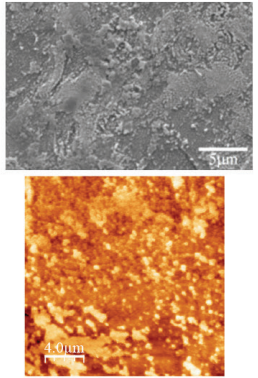

(b)

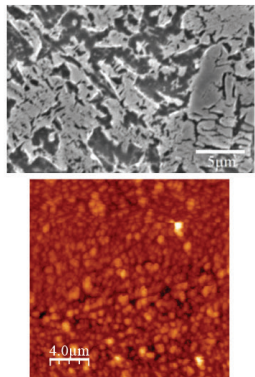

(c)

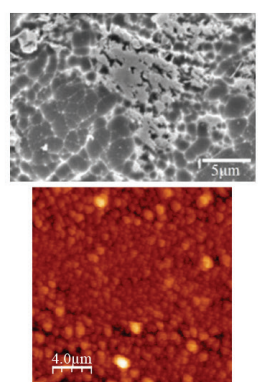

(d)

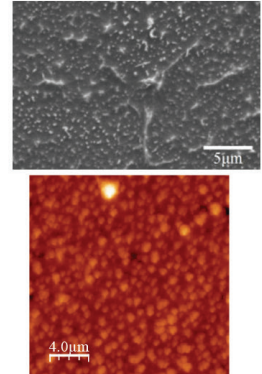

(e)

Figure 3. SEM images (top) $(8 \mathrm{kV} ; \times 5.000)$ and AFM micrpgraphs (bottom) $(20 \mu \mathrm{m} \times 20 \mu \mathrm{m})$ of polymer samples treated by DBD non-thermal plasma. (A) PP (adapted from reference 35); (B) HDPE; (C) PA6.6; (D) PEEK. (a) Non treated; (b) 5 min; (c) 20 min; (d) 60 min; (e) 100 min.

due to its lower crystallinity and density when compared with PEEK.

Considering the chemical structure of PEEK, it is not expected to undergo crosslinking or be easily degraded, since the structure is based on aromatic rings which act as an energy sink, protecting the organic material. In addition, PEEK exhibits one of the highest values for abrasion resistance and a density greater than $1.26 \mathrm{~g} \mathrm{~cm}^{-3}$. According to Hattori and Itoh, ${ }^{44}$ the resistance to cavitation, abrasion and mechanical stress is related to the density of 

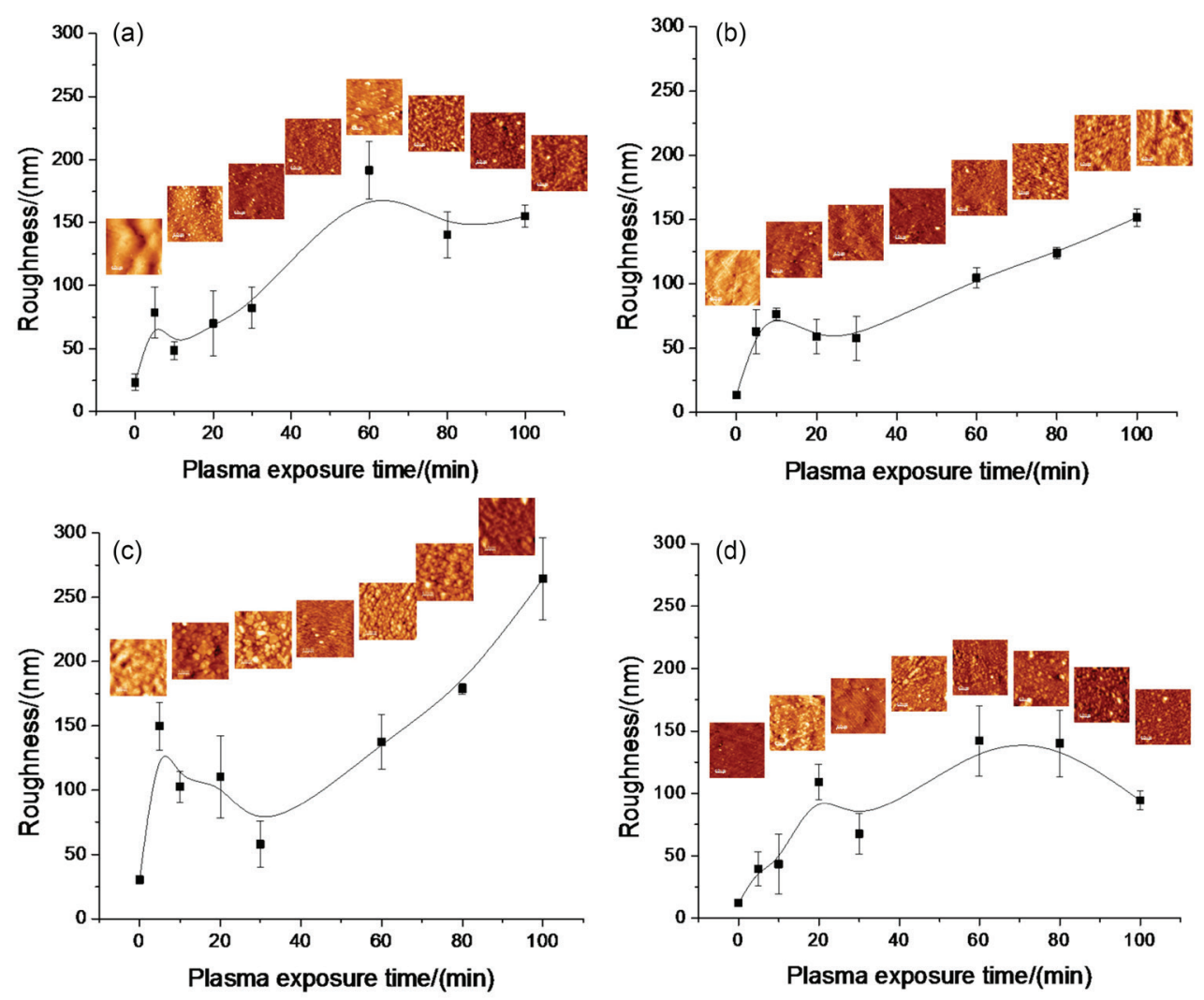

Figure 4. Temporal evolution of roughness (nm) for different polymers exposed to NTP. The lateral size of the samples in the micrographs was $20 \mu \mathrm{m}$. (a) PP; (b) HDPE; (c) PA6.6; (d) PEEK.

both metallic and polymeric materials, as well as other types of materials.

The main process associated with the application of NTP to polymeric surfaces is the occurrence of gradual etching, which eliminates volatile fragments and causes morphological changes in the material. Exposure of the polymer to plasma discharge is also associated with the crosslinking effect, which occurs simultaneously with depolymerization, oxidation, degradation and the formation of $\mathrm{CO}_{2}, \mathrm{CO}$ and $\mathrm{H}_{2} \mathrm{O}$.

The crosslinking is basically promoted by UV radiation and occurs immediately in the surface layer reaching a few micrometers inside the bulk of the material. ${ }^{43}$ In relation to sputter etching, polymers can be classified according to their susceptibility to reorder into reticulated structures, to form crosslinking, or to degrade into smaller fragments (scission). ${ }^{45-48}$ The formation of crosslinking converts a linear polymer into a three-dimensional molecule, resulting in an increase and often immeasurable molecular mass, and usually improving the mechanical properties ${ }^{45,47}$ and resistance to degradation. ${ }^{49}$ In this sense, polymers with different structure and functional groups define the resistance to chemical processes to which they will be subjected. In an atmosphere of ionizing radiation, for instance, aromatic and conjugated systems present greater resistance due to the absorption of photons or the transfer of energy or charged particles. On the other hand, aliphatic chains undergo hydrogen abstraction, leaving $\mathrm{C}^{\cdot}$ radical sites, which easily result in oxidation, double bond formation, degradation and crosslinking sites. ${ }^{42}$

Figure 4 shows the temporal evolution of the roughness development (nm) of polymers surfaces exposed to NTP where a two-step processes can be identified. The first step begins immediately after the onset of exposure and continues up to 20-30 min, for all polymers. The second step is then evident in the case of PP and PEEK. Both HDPE and PA6.6 present homogeneous growth with only one step of decreasing roughness. This small surface fraction $(100-150 \mathrm{~nm})$ seems to be more susceptible to plasma species, since in the first few minutes it presents an abrupt increase in the roughness, estimated by the slope of the initial portion of the curve.

As described by the cascade collision model of Hoogebrugge and Kistermaker, ${ }^{50}$ it is clearly perceived for all polymers, that during the initial moments of the NTP exposure there is an abrupt increase in the roughness, which reaches a threshold (atomic collisions). After this point, the ions tend to penetrate deeper into the material, decreasing the energy deposited in the outermost layer. This energy at greater depth initiates the etching and the roughness 
grows almost linearly over time, for the majority of the samples studied. ${ }^{49}$

The general behavior of the curves shown in Figure 4 can be analyzed based on steps of increasing and decreasing surface roughness, which have direct relationship with the formation of peaks and valleys, since plasma continuously provide active/reactive species.

The first step, from 0 to $30 \mathrm{~min}$, the NTP treatment induces the increase and decrease of surface roughness related to the formation of peaks and wear of the peaks. The second step is easily observed for PP and PEEK shown in Figures 4a and 4d, respectively, with an increase and decrease of surface roughness, however for PA6.6 and HDPE the second step has not been reached with the surface roughness that is still growing.

Figure 5 shows the roughness values $(\mathrm{nm})$ of three scanned regions (areas A, B and C) as a function of scan size analyzed for the neat PP (Figure 5a) and after exposure to the plasma for $5 \mathrm{~min}$ (Figure $5 \mathrm{~b}$ ). In each region were obtained three area sizes. In Figure 5a the roughness decreases when the scan size is magnified, i.e., when the size of area decreases in the same region. This means that there is a global roughness with large clusters to major area and a local roughness to small area of scan without NTP. The Figure $5 b$ shows that the roughness values to PP polymer during $5 \mathrm{~min}$ of NTP are approximately constant for different areas and scan size. The NTP etches hard sites leaving more homogeneous surface. Statistically, these differences are important and must be taken into account. However, the analysis of the log-data from which the exponents were obtained presents a smaller variation.

The roughness data for all polymers sample were obtained using the same experimental procedure and acquired in function of the size of the scanned area and the time of exposure to the NTP. These data were then used in the statistical calculations. This information can be found in detailed in the SI section (Figures S1-S4).

According to Figure $6 \mathrm{a}$ the roughness exponent values were around $0.70<\alpha<0.76$ for the polymers, considering the associated errors. Although similar, the values of the
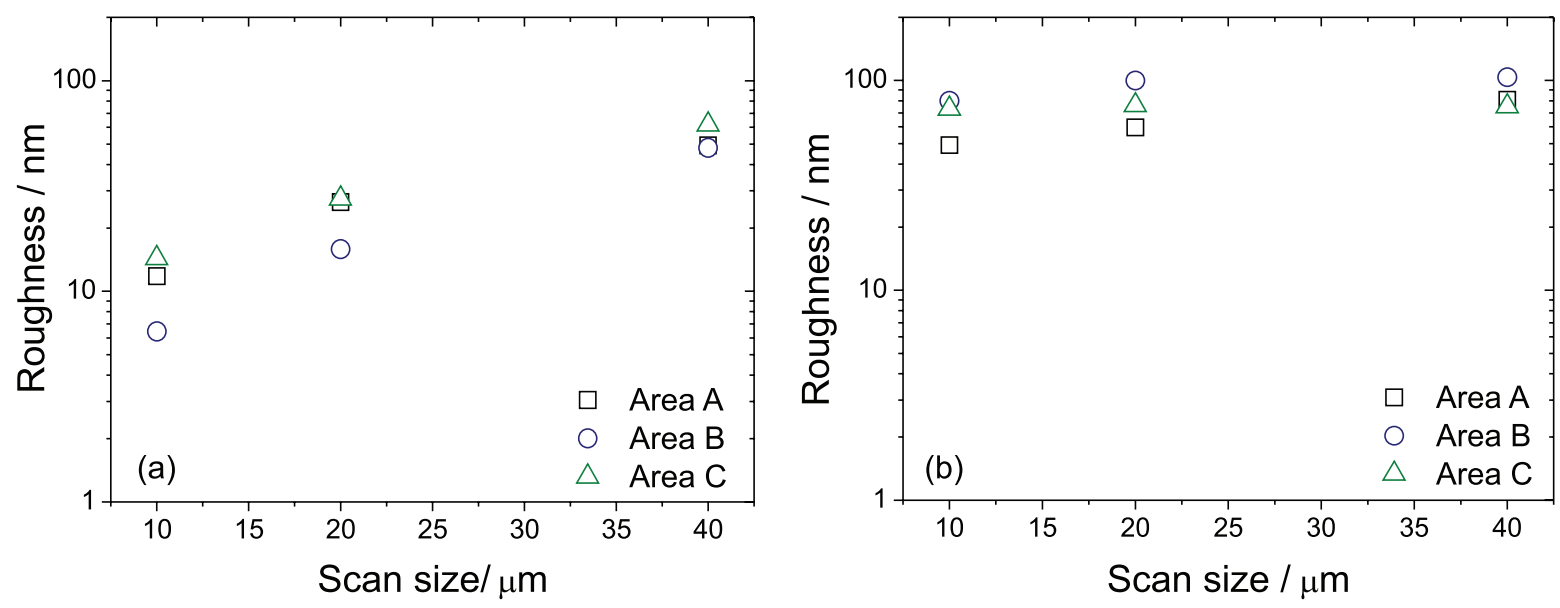

Figure 5. Graphical demonstration of roughness (nm), as a function of scan size ( $\mu \mathrm{m})$, for the pure PP (a) and the sample exposed to the NTP for 5 min (b).
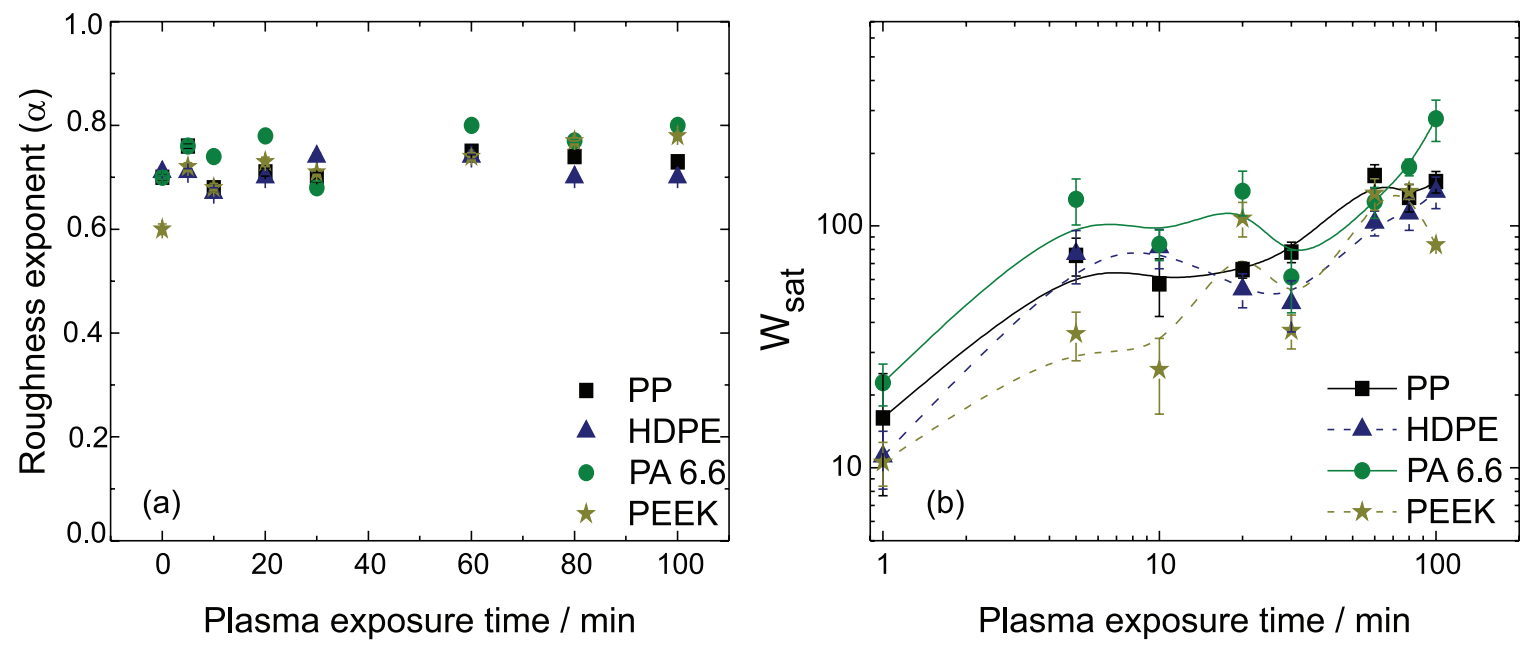

Figure 6. (a) Profiles for the roughness exponent and (b) log-log plot of the saturation roughness as a function of the NTP exposure time. 
roughness exponents for PA6.6 and PEEK, especially for high exposure time, were slightly larger than for PP and HDPE. In these intervals, it can be assumed that the surfaces are momentarily less irregular, i.e., the morphology presents lower fluctuations of high frequency.

Despite the differences in average roughness presented in Figure 4, the roughness exponent (Figure 6a) was similar for all polymers studied, showing that the growth model is a particular characteristic of these materials.

The saturation values $\left(\mathrm{W}_{\text {sat }}\right)$ as a function of the exposure time, as well as the roughness profile, show that the surface growth is characterized by successive slopes in a non-linear dynamic behavior, as shown in Figure 6b. These profiles show that the growth occurs in a correlated way and that the surface formation process is uniform and similar for all polymers. These profiles provide $\beta$ values of $0.84<\beta<1.2$. Table 3 lists the critical exponents obtained from the statistical treatment of all polymers tested.

Table 3. Roughness and growth exponent data for polymers exposed to NTP

\begin{tabular}{lcc}
\hline Polymer & $\alpha$ & $\beta$ \\
\hline PP & 0.73 & 0.93 \\
HDPE & 0.71 & 1.2 \\
PA6.6 & 0.76 & 1.06 \\
PEEK & 0.72 & 0.84 \\
\hline
\end{tabular}

$\alpha$ : roughness exponent; $\beta$ : growth exponent. PP: polypropylene; HDPE: high density polyethylene; PA6.6: polyamide 6.6; PEEK: poly(ether ether) ketone.

Therefore, the mean values for the exponents determined for the polymers from the experimental data were: $\alpha$ ca. 0.73 and $\beta$ ca.1.0, for all samples studied. The statistical data for the surfaces of the PP, HDPE, PA6.6 and PEEK samples studied herein are consistent with the self-affine behavior. The parameters found by Agarwal et al. ${ }^{31}$ of $\alpha$ ca. $0.6 \pm 0.1$ and $\beta$ ca.1.0, for a polyimide are similar to the results reported herein. Furthermore, the cited authors observed that the roughness had a non-linear behavior at the beginning of the exposure.

As previously mentioned, some models proposed in the literature ${ }^{31}$ provide similar results to those obtained in this study, with respect to the exponent growth, $\beta$. Considering the randomness of sputter etching and the continuous feeding of active species in the characteristic NTP process, we can conclude that there is a synergic relationship between the "shadowing growth" proposed by Pétri et al. ${ }^{28}$ and the redistribution of the active species, proposed by Drotar et al. ${ }^{30}$ In this study, we associate the stages of surface relaxation and momentary flattening with the increased density due to crosslinking caused by exposure to UV radiation promoted by the plasma discharge.

Figure 7 shows the dependence of the correlation length (Lc) on the plasma exposure time for all samples. It can be noted that, in the case of PP, the maximum $\mathrm{Lc}$ value was obtained with no plasma exposure. This value was much higher than those for the other samples and it decreased with the etching time only from 0 to $10 \mathrm{~min}$, and then remained constant until the end of the exposure period. This constancy was also observed for the other samples, where Lc remained almost unchanged. Except for the PP sample without plasma treatment, the saturation correlation length between the self-affine and saturation region was approximately $1.2 \pm 0.2 \mu \mathrm{m}$ for all samples, which means that, for distances larger than $1.2 \mu \mathrm{m}$ the surfaces are completely correlated. For the PP sample, with no plasma etching, Lc was around $5 \mu \mathrm{m}$ (the highest value obtained) probably due to the experimental procedure applied during its preparation. The saturation values $\left(\mathrm{W}_{\text {sat }}\right)$ as a function of Lc, for all polymers, can be consulted in the SI section (Table S2).

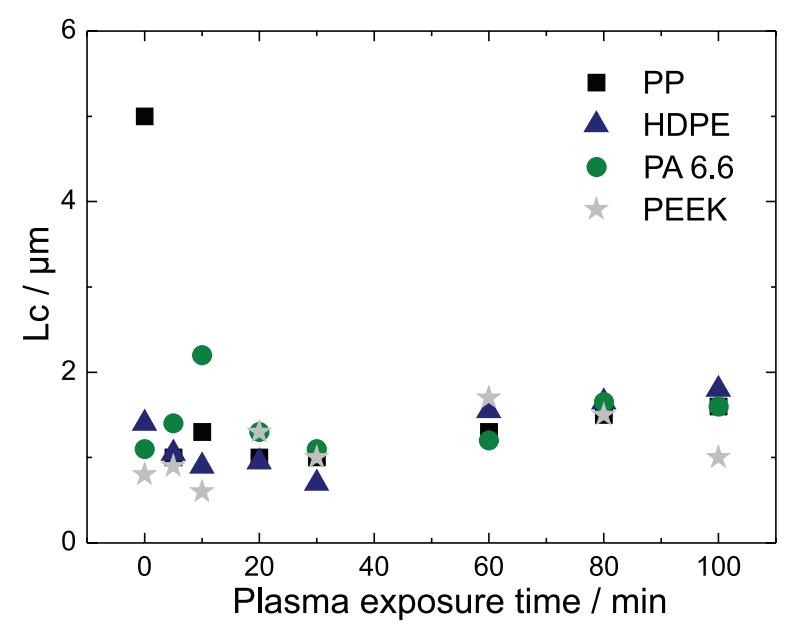

Figure 7. Correlation length (Lc) as a function of the plasma exposure time for all the samples analyzed.

\section{Conclusions}

Based on the properties of the polymers investigated in this study, the crosslinking effect appears to be mainly responsible for the observed rigidity and resistance to bombardment by the species originating from the plasma source. The sputter etching of the surface would preferably act on spots of less physical rigidity or non-crosslinked. These "rigid" sites require more energy to be eroded and are not easily removed from the surface, and are considered quenched noise in the scaling law. ${ }^{32}$ This hypothesis can be explained by the successive decreases and increases in the roughness observed for the studied materials. In addition, it 
is possible to classify the HDPE and PP as more vulnerable to the chain scission and crosslinking effect, respectively, while the PA6.6 is more susceptible to fragmentation than the previously mentioned materials, probably because it contains - NCO- and-CO- groups. Also, PEEK was more resistant to the species generated by the plasma compared with the other polymers, and the effects of crosslinking or fragmentation were not observed due to its chemical composition.

The saturation values $\left(\mathrm{W}_{\text {sat }}\right)$ and roughness profile shows that the growth occurs in a correlated way and that the surface formation process is similar for all polymers. The values of the roughness and growth exponents obtained for the polymers exposed to the NTP were $\alpha$ ca. $0.73 \pm 0.2$ and $\beta$ ca. $1.0 \pm 0.1$, which are considered practically unchanged and independent of the specific properties of each material. The statistical data suggests consistency with the self-affine behavior.

Recent studies have provided a reasonable approximation of the mechanism that governs sputter etching and surface growth where the models of shadowing and redistribution of active species were considered as the dominant synergistic effects governing the process. Additionally, both the stages of surface relaxation and momentary flattening have been associated with the increased density due to crosslinking caused by exposure to UV radiation promoted by nonthermal plasma.

Knowledge of the growth process of plasma-treated polymers surfaces, regardless of the electrical parameters, allows an estimation of the time required to reach a certain morphological condition, making this study of great industrial interest. The exponents obtained and their small variation for the different polymers demonstrates that the roughness variable can be controlled in a satisfactory way.

\section{Supplementary Information}

The authors provide the supplementary information about chemical composition of polymers, based on $\mathrm{C} 1 \mathrm{~s}$ and $\mathrm{O} 1 \mathrm{~s}$ groups, details on the acquisition of roughness values according to the size samples and other information necessary for statistical treatment based on scaling laws. Supplementary information (Tables S1 and S2, Figures S1-S4) is available free of charge at http://jbcs.sbq.org.br as PDF file.

\section{Acknowledgments}

T. Felix would like to thank the doctoral programs of Science and Engineering Materials of Faculdade de Ciências e Tecnologia da Universidade Nova de Lisboa,
Cenimat/i3N, Chemistry Department of Federal University of Santa Catarina and Erasmus Mundus Programme for the opportunity to carry out a sandwich doctorate. The authors also acknowledge the financial support from FEDER, through Programa Operacional Factores de Competitividade (COMPETE), Fundação para a Ciência e a Tecnologia (FCT), for the project UID/FIS/00068/2013 and the Brazilian agencies: CNPq and CAPES/PRINTUFSC, 2019.

\section{References}

1. Meakin, P.; Fractals, Scaling and Growth far from Equilibrium; Cambridge University Press: Cambridge, United Kingdom, 2011.

2. Motojima, S.; Itoh, C.; Iwanaga, H.; J. Mater. Sci. 1991, 26, 1467.

3. Mandelbrot, B. B.; Wheeler, J. A.; Am. J. Phys. 1983, 51, 286.

4. Constantoudis, V.; Patsis, G. P.; Gogolides, E.; Microelectron. Eng. 2012, 90, 121.

5. Johnson, D.; Hilal, N.; J. Membr. Sci. 2019, 570-571, 9.

6. Ostrikov, K.; Vacuum 2008, 83, 4.

7. Prähofer, M.; Spohn, H.; J. Stat. Phys. 2004, 115, 255.

8. Forgerini, F. L.; Marchiori, R.; Biomatter 2014, 4, e28871.

9. Zekonyte, J.; Zaporojtchenko, V.; Faupel, F.; Nucl. Instrum. Methods Phys. Res., Sect. B 2005, 236, 241.

10. Barabasi, A.-L.; Stanley, H. E.; Fractal Concepts in Surface Growth; Cambridge University Press: New York, 1995.

11. Edwards, S. F.; Wilkinson, D. R.; Proc. R. Soc. London, Ser. A 1982, A381, 504.

12. Dotto, M.; Kleinke, M.; Phys. Rev. B 2002, 65, 245323.

13. Gabrielli, A.; Baldassarri, A.; Sapoval, B.; Phys. Rev. E: Stat. Phys., Plasmas, Fluids, Relat. Interdiscip. Top. 2000, 62, 3103.

14. Faita, F. L.; Dotto, M. E. R.; França, L. G.; Cabrera, F. C.; Job, A. E.; Bechtold, I. H.; Eur. Polym. J. 2014, 50, 249.

15. Yadav, R. P.; Kumar, M.; Mittal, A. K.; Dwivedi, S.; Pandey, A. C.; Mater. Lett. 2014, 126, 123.

16. Dotto, M. E. R.; Martins, R. N.; Ferreira, M.; Camargo, S. S.; Surf. Coat. Technol. 2006, 200, 6479.

17. Allongue, P.; Kasparian, J.; Elwenspoek, M.; Surf. Sci. 1997, 388,50 .

18. Santra, S. B.; Sapoval, B.; Phys. A. 1999, 266, 160.

19. Brault, P.; Dumas, P.; Salvan, F.; J. Phys.: Condens. Matter 1998, 10, L27.

20. Martin, M.; Cunge, G.; J. Vac. Sci. Technol., B: Microelectron. Nanometer Struct.--Process., Meas., Phenom. 2008, 26, 1281.

21. Bae, J.; Lee, I. J.; Sci. Rep. 2015, 5, 10126.

22. Pani, S. K.; Wong, C. C.; Sudharsanam, K.; Premachandran, C. S.; Iyer, M. K.; J. Vac. Sci. Technol., B: Microelectron. Nanometer Struct.--Process., Meas., Phenom. 2006, 24, 163. 
23. Bruce, R. L.; Weilnboeck, F.; Lin, T.; Phaneuf, R. J.; Oehrlein, G. S.; Long, B. K.; Willson, C. G.; Vegh, J. J.; Nest, D.; Graves, D. B.; J. Appl. Phys. 2010, 107, 084310.

24. Vourdas, N.; Kontziampasis, D.; Kokkoris, G.; Constantoudis, V.; Goodyear, A.; Tserepi, A.; Cooke, M.; Gogolides, E.; Nanotechnology 2010, 21, 085302.

25. Kontziampasis, D.; Constantoudis, V.; Gogolides, E.; Plasma Processes Polym. 2012, 9, 866.

26. Kokkoris, G.; Constantoudis, V.; Angelikopoulos, P.; Boulousis, G.; Gogolides, E.; Phys. Rev. B: Condens. Matter Mater. Phys. 2007, 76, 193405.

27. Kokkoris, G.; Gogolides, E.; J. Phys. D: Appl. Phys. 2012, 45, 165204.

28. Pétri, R.; Brault, P.; Vatel, O.; Henry, D.; André, E.; Dumas, P.; Salvan, F.; J. Appl. Phys. 1994, 75, 7498.

29. Zhao, Y. P.; Drotar, J. T.; Wang, G. C.; Lu, T. M.; Phys. Rev. Lett. 1999, 82, 4882.

30. Drotar, J.; Zhao, Y.-P.; Lu, T.-M.; Wang, G.-C.; Phys. Rev. B 2000, 61, 3012.

31. Agarwal, N.; Ponoth, S.; Plawsky, J.; Persans, P. D.; Appl. Phys. Lett. 2001, 78, 2294.

32. Buldyrev, S. V.; Barabási, A.; Caserta, L. F.; Havlin, S.; Stanley, R. E.; Vicsek, T.; Phys. Rev. A 1992, 45, R8313(R).

33. Mark, J. E.; Polymer Data Handbook; Oxford University Press: Oxford, 1999.

34. Teodoro, O.; Silva, J.; Moutinho, A.; Vacuum 1995, 46, 1205.

35. Felix, T.; Trigueiro, J. S.; Bundaleski, N.; Teodoro, O. M. N. D.; Sério, S.; Debacher, N. A.; Appl. Surf. Sci. 2018, 428, 730.

36. Horcas, I.; Fernández, R.; Gómez-Rodríguez, J. M.; Colchero, J.; Gómez-Herrero, J.; Baro, A. M.; Rev. Sci. Instrum. 2007, 78,013705 .
37. Felix, T.; Cassini, F. A.; Benetoli, L. O. B.; Dotto, M. E. R.; Debacher, N. A.; Appl. Surf. Sci. 2017, 403, 57.

38. Kostov, K. G.; Nishime, T. M. C.; Castro, A. H. R.; Toth, A.; Hein, L. R. O.; Appl. Surf. Sci. 2014, 314, 367.

39. Oehrlein, G. S.; Phaneuf, R. J.; Graves, D. B.; J. Vac. Sci. Technol., B: Microelectron. Nanometer Struct.--Process., Meas., Phenom. 2011, 29, 010801.

40. Puliyalil, H.; Cvelbar, U.; Nanomaterials 2016, 6, 108.

41. Fridman, A.; Plasma Chemistry; Cambridge University Press: Cambridge, 2008.

42. Friedrich, J. F.; The Plasma Chemistry of Polymer Surfaces; Wiley-VCH: Weinheim, Germany, 2012.

43. Friedrich, J.; Kuhn, G.; Gahde, J.; Acta Polym. 1979, 30, 470.

44. Hattori, S.; Itoh, T.; Wear 2011, 271, 1103.

45. Woods, R. J.; Pikaev, A. K.; Applied Radiation Chemistry: Radiation Processing; John Wiley and Sons: New York, 1994.

46. Taylor, G.; Wolf, T. M.; Polym. Eng. Sci. 1980, 20, 1087.

47. Chapiro, A.; Radiation Chemistry of Polymeric Systems; Interscience Publischers: New York, 1962.

48. Schnabel, W.; Polymer Degradation: Principles and Practical Applications; Hanser International: Munich, Germany, 1981.

49. Zekonyte, J.: Sputtering and Surface Modification of Thermoplastic Polymers with Low Energy Ion Beams; MSc Dissertation, Christian-Albrechts Universitat, Kiel, Germany, 2005, available at https://macau.uni-kiel.de/receive/diss_ mods_00001492?lang=en, accessed in May 2020.

50. Hoogebrugge, R.; Kistermaker, P. G.; Nucl. Instrum. Methods Phys. Res., Sect. B 1987, 21, 37.

Submitted: January 8, 2020 Published online: May 27, 2020 